\section{Neuer VEGF-Inhibitor verlängert Leben nicht}

\author{
Bei den meisten Patienten mit nichtkleinzelligem Lungenkarzinom (NSCLC) \\ kommt es nach der First-Line-Therapie zur Progression. Nun wurde mit \\ Aflibercept eine neue Option für die Second-Line geprüft.
}

\begin{abstract}
A flibercept ist ein rekombinantes humanes Fusionsprotein, das den Signalweg des vaskulären endothelialen Wachstumsfaktors (VEGF) hemmt. In der VITAL-Studie wurde die Wirksamkeit von Aflibercept bei Patienten mit fortgeschrittenem oder metastasiertem, nichtsquamösem NSCLC nach der platinbasierten First-Line-Therapie überprüft. In dieser doppelblinden, placebokontrollierten, randomisierten PhaseIII-Studie erhielten 913 Patienten entweder Aflibercept $(6 \mathrm{mg} / \mathrm{kg})$ oder Placebo i.v. alle drei Wochen in Kombination mit Docetaxel $\left(75 \mathrm{mg} / \mathrm{m}^{2}\right)$. Primärer Endpunkt war das Gesamtüberleben.

Aflibercept verbesserte das Gesamtüberleben nicht (Hazard Ratio [HR] 1,01;
\end{abstract}

log-rank $\mathrm{p}=0,90)$. Das mediane Gesamtüberleben betrug 10,1 Monate unter Aflibercept und 10,4 Monate in der Placebo-Gruppe. Das berechnete 1-JahresÜberleben betrug in beiden Gruppen $45 \%$. Die Ergebnisse waren über die meisten Subgruppen konsistent. Auch eine vorherige Therapie mit Bevacizumab (12,3\% der Patienten) hatte keinen Einfluss.

In den sekundären Endpunkten hatte Aflibercept jedoch signifikante Vorteile: Es verlängerte das progressionsfreie Überleben (5,2 vs. 4,1 Monate; HR 0,82; $\mathrm{p}=0,0035)$ und erhöhte die Gesamtansprechrate ( 23,3 vs. $8,9 \%$; $<<0,001)$.

Allerdings ging die Therapie mit Aflibercept mit mehr Nebenwirkungen ein- her, wie sie von anderen Anti-VEGFund Chemotherapien bekannt sind.

Unerwünschte Ereignisse vom Grad 3 oder höher traten unter Aflibercept plus Docetaxel bei 71,5\% der Patienten auf, unter Docetaxel allein dagegen „nur" bei $49,7 \%$. Am häufigsten entwickelten die Patienten eine Neutropenie (28,0 vs. $21,1 \%$ ), Fatigue (11,1 vs. $4,2 \%$ ), Stomatitis $(8,8$ vs. $0,7 \%)$ und Hypertonie ( 7,3 vs. $0,9 \%)$.

Fazit: Bei NSCLC-Patienten verlängerte die Zweitlinienbehandlung mit Aflibercept zusätzlich zur Standardtherapie mit Docetaxel das Gesamtüberleben nicht. Verbesserungen zeigten sich aber in sekundären Endpunkten wie progressionsfreiem Überleben und Gesamtansprechen.

Judith Neumaier

Ramlau R et al. Aflibercept and docetaxel versus docetaxel alone after platinum failure in patients with advanced or metastatic non-smallcell lung cancer: A randomized, controlled phase III trial. J Clin Oncol. 2012; 30(29):3640-7.

\section{Besser als Abwarten: Erhaltungstherapie lohnt sich}

\begin{abstract}
Abwarten und beobachten war bisher die Strategie der Wahl bei Patienten mit nichtkleinzelligem Lungenkarzinom (NSCLC) im Stadium IV nach der First-Line-Chemotherapie. Seit der Einführung neuer, besser verträglicher Medikamente scheint jedoch eine Erhaltungstherapie sinnvoller zu sein.
\end{abstract}

Er üher war die Verlängerung der platinbasierten First-Line-Therapie bei Patienten mit NSCLC wegen kumulativer Toxizitäten kaum durchführbar. Seit besser verträgliche Medikamente verfügbar sind, ist die Erhaltungstherapie eine realisierbare Option. Zwei Strategien der Erhaltungstherapie sind möglich: Die Fortführung der Nicht-Platin-Komponente der First-Line-Therapie und der Umstieg auf ein neues Medikament.

In der Phase-III-Studie IFCT-GFPC 0502 wurden diese Strategien bei $464 \mathrm{~Pa}-$ tienten mit NSCLC im Stadium IIIB-IV, das nach vier Zyklen einer Induktionschemotherapie mit Cisplatin und Gemcitabin nicht progredient war, untersucht. Die Patienten wurden in drei Gruppen randomisiert: Beobachtung, weitere Gabe von Gemcitabin $\left(1.250 \mathrm{mg} / \mathrm{m}^{2}\right.$ an den Tagen 1 und 8, q3w) oder Weiterbe- handlung mit Erlotinib (150 mg/Tag). Kam es zur Progression, erhielten die Patienten in allen drei Armen Pemetrexed $\left(500 \mathrm{mg} / \mathrm{m}^{2}, \mathrm{q} 3 \mathrm{w}\right)$ in der Second-Line.

Beim progressionsfreien Überleben, dem primären Endpunkt der Studie, waren beide Erhaltungstherapien der alleinigen Beobachtung signifikant überlegen: Sowohl Gemcitabin (median 3,8 vs. 1,9 Monate; Hazard Ratio [HR] 0,56; $\mathrm{p}<$ 0,001 ) als auch Erlotinib (median 2,9 vs. 1,9 Monate; HR 0,$69 ; p=0,003$ ) verlängerten das progressionsfreie Überleben. Der Nutzen der Erhaltungstherapie war in allen Subgruppen (Raucherstatus, Histologie, Geschlecht, EGFR-Expression) konsistent. Allerdings gab es einen Trend zu einem größeren Nutzen der Erhaltungstherapien bei einer SecondLine-Therapie mit Pemetrexed. Außerdem war der Nutzen von Gemcitabin tendenziell höher bei Patienten, die objektiv auf Cisplatin/Gemcitabin angesprochen hatten, der von Erlotinib bei Nichtrauchern.

Auf das Gesamtüberleben hatten beide Erhaltungstherapien keinen signifikanten Einfluss. Allerdings könnte das Ansprechen auf die Induktionschemotherapie sich positiv auf das Gesamtüberleben nach Gemcibatin-Erhaltungstherapie auswirken.

Gemcitabin und Erlotinib waren in der Erhaltungstherapie gut verträglich, es traten keine unerwarteten unerwünschten Ereignisse auf.

Fazit: Mit einer Erhaltungstherapie mit Gemcitabin oder Erlotinib bleiben Patienten mit fortgeschrittenem NSCLC, die in der First-Line Cisplatin und Gemcitabin erhalten hatten, signifikant länger progressionsfrei.

Judith Neumaier

Pérol M et al. Randomized, phase III study of gemcitabine or erlotinib maintenance therapy versus observation, with predefined secondline treatment, after cisplatin-gemcitabine induction chemotherapy in advanced nonsmall-cell lung cancer. J Clin Oncol. 2012; 30(28):3516-24. 